\title{
Myocardial Stunning Caused by Sympathetic Nerve Injury After an Operation on Cervical Vertebrae
}

\author{
Shuji Hatakeyama, MD; Yoshinori Oda, MD*; \\ Tadahiro Kurasawa, MD*; Shinichi Kimata, MD*
}

\begin{abstract}
In this case, electrocardiographic inverted $\mathrm{T}$ waves appeared after cervical laminaplasty and echocardiogram showed temporary wall motion abnormality. Myocardial metaiodobenzylguanidine (MIBG) uptake was obviously reduced in the same area where the wall motion abnormalities appeared in the echocardiogram, although no abnormalities were detected with myocardial thallium scintigraphy and coronary angiography. The myocardial stunning was caused by injury to the sympathetic nerves from a surgical procedure on the cervical vertebrae. (Jpn Circ J 1999; 63: 216-218)
\end{abstract}

Key Words: Laminaplasty; Metaiodobenzylguanidine (MIBG) scintigraphy; Myocardial stunning; Sympathetic nervous system; T-wave inversion

$\mathbf{M}$ yocardial stunning is described as prolonged but reversible myocardial contractile dysfunction of previously ischemic myocardium salvaged by coronary reperfusion therapy and bypass surgery. Furthermore, in recent reports myocardial stunning has been observed in patients suffering cerebrovascular attack or after electroconvulsive therapy, and the sympathetic nervous system is suspected to have some relationship to the cause. The electrocardiographic inverted $\mathrm{T}$ wave detects the existence of ischemic heart disease, but an electrolyte imbalance or various other incidents may also induce the electrocardiographic $\mathrm{T}$ wave inversion. In patients with cerebrovascular disease, similar ST-T wave changes are sometimes observed, and the cause of such changes is considered to be based on sympathetic nervous disorders.

A case is presented of myocardial stunning and drastic ST-T wave changes caused by injury of the sympathetic nerves from a surgical procedure on the cervical vertebrae.

\section{Case Report}

A 77-year-old woman was performed for laminaplasty at the $\mathrm{C} 3-5$ spine level for $\mathrm{C} 3 / 4$ disk herniation and ossification of the posterior longitudinal ligament (OPLL). Her electrocardiogram on admission was within normal limits except for a flat $\mathrm{T}$ wave in leads of II, III, $\mathrm{aVF}$ and $\mathrm{V}_{6}$. In the day following the operation, such drastic electrocardiographic changes as inverted $\mathrm{T}$ wave in $\mathrm{V}_{1-6}$ and prominent QT interval prolongation were revealed (Fig 1). The electrocardiographic changes illustrated a strong similarity to that of subendocardial myocardial infarction, but any cardiovascular symptoms were absent.

(Received September 11, 1998; revised manuscript received November 16, 1998; accepted December 4, 1998)

Department of Internal Medicine, Showa General Hospital and *Department of Internal Medicine, Tokyo Kousei-Nenkin Hospital, Tokyo, Japan

Mailing address: Shuji Hatakeyama, MD, Department of Internal Medicine, Showa General Hospital, 2-450, Tenjincho, Kodaira-City, Tokyo 187-8510, Japan
She had no history referable to a cardiovascular system disorder, and no coronary risk factors such as smoking, diabetes mellitus or hypertension. Her body temperature then was $37.1^{\circ} \mathrm{C}$ and blood pressure was $110 / 58 \mathrm{mmHg}$, with no remarkable changes in her vital or physical signs. Laboratory data were as follows: aspartate aminotransferase (GOT) $44 \mathrm{IU} / \mathrm{l}$, alanine aminotransferase (GPT) 25 $\mathrm{IU} / \mathrm{L}, \mathrm{Na} 135 \mathrm{mmol} / \mathrm{L}, \mathrm{K} 3.8 \mathrm{mmol} / \mathrm{L}, \mathrm{Cl} 98 \mathrm{mmol} / \mathrm{L}$, creatime kinase 464 IU/L (MM 98\%, MB 2\%), lactate dehydrogenase $183 \mathrm{IU} / \mathrm{L}$, C-reactive protein $7.35 \mathrm{mg} / \mathrm{dl}$, white blood cell count $8.0 \times 10^{3} \mu \mathrm{l}$, hemoglobin $11.0 \mathrm{~g} / \mathrm{dl}$, hematocrit $32.7 \%$, platelets $24.6 \times 10^{4} \mu \mathrm{l}$, myosin light chain $16 \mathrm{ng} / \mathrm{ml}$, and troponin T $0.22 \mathrm{ng} / \mathrm{ml}$.

An echocardiogram revealed left ventricle apex hypokinesis with a full thickness myocardium, but that obtained 11 days after the operation demonstrated almost complete recovery with no wall motion abnormalities. ${ }^{123} \mathrm{I}-$ metaiodobenzylguanidine(MIBG) and thallium-201 $\left({ }^{201} \mathrm{Tl}\right)$ dual scintigraphic assessment of her myocardium function were performed on the 5th postoperative day. MIBG accumulation was obviously reduced in the apex-anterior left ventricle, but the thallium perfusion image was otherwise homogeneous (Fig 2).

On the 25th postoperative day, coronary arteriography was performed. No stenosis was detected, and no coronary vasospasm was induced by intracoronary administration of acetylcholine. Further, she was not taking reserpine, tricyclic antidepressants or any other drugs that would interfer with the uptake of MIBG.

\section{Discussion}

In this case, the initial ECG change and the appearance of echocardiographic myocardial hypokinesis after operation on the cervical vertebrae were thought to be changes due to subendocardial myocardial infarction. However, the lack of any cardiovascular symptoms, the absence of any rise in serum myocardial enzymes except for a slight elevation of serum myosin light chain, and the absence of any history referable to a cardiovascular system disorder made it less likely. Further, she had no coronary stenosis and no 


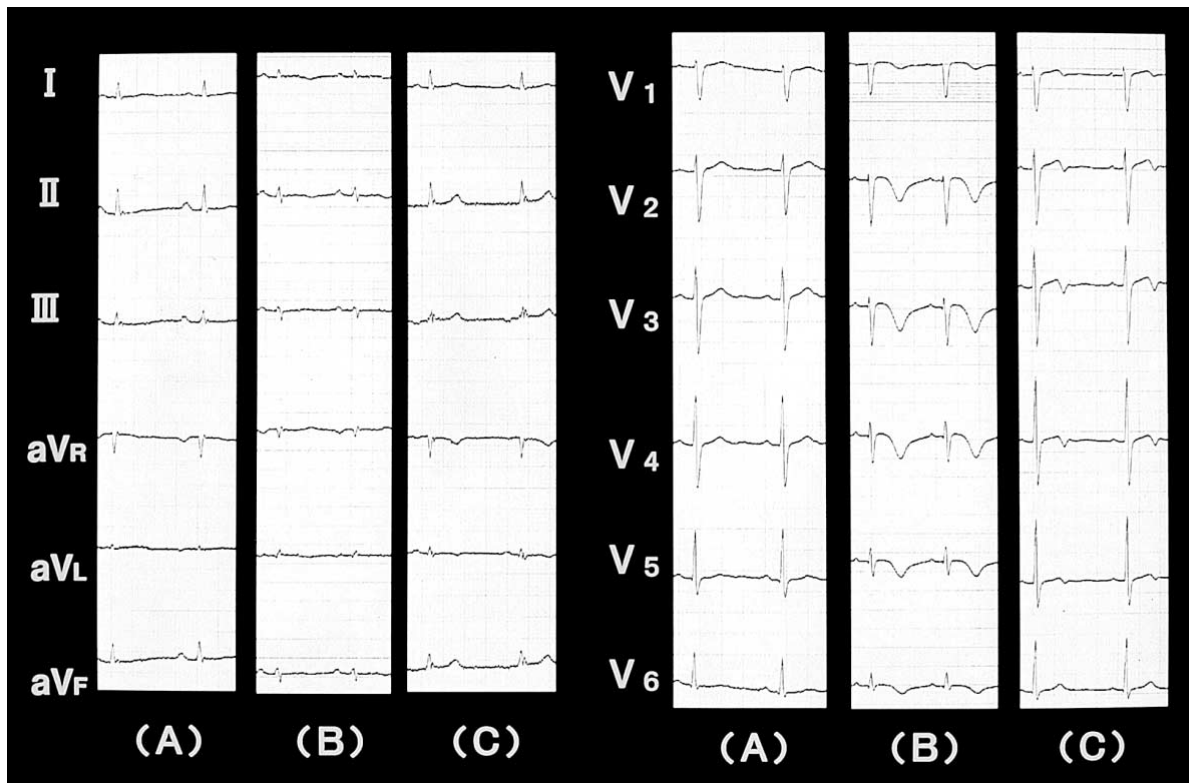

Fig 1. Electrocardiogram on admission (A), the day after the operation (B) and on the 45th day (C). Drastic electrocardiographic changes such as inverted $\mathrm{T}$ wave in $\mathrm{V}_{1-6}$ and prominent QT interval prolongation were revealed.

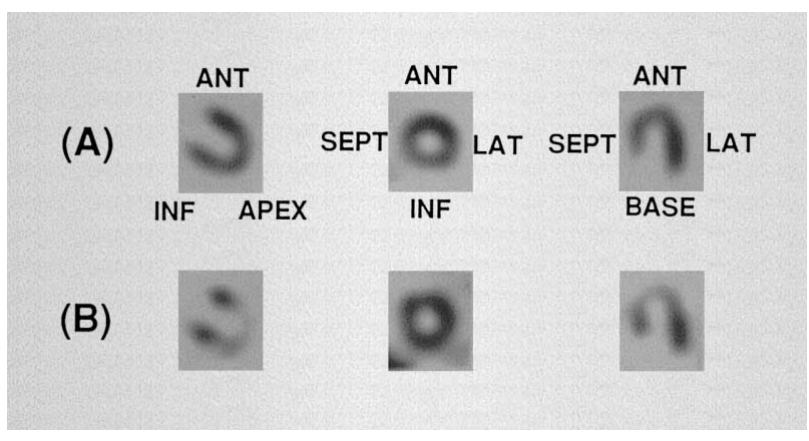

Fig 2. MIBG images are in the lower rows (B), and simultaneously acquired thallium images are in the upper (A). MIBG accumulation was reduced in the apex-anterior of the left ventricle, whereas the thallium perfusion image was homogeneous.

defect on Tl-scintigraphy. MIBG accumulation was obviously reduced in the apex-anterior left ventricle just as hypokinesis was observed in the same location on echocardiography, so the existence of some sympathetic disorder was suspected in that area. A few weeks later, the myocardial wall motion abnormality returned to normal, and electrocardiographic $\mathrm{T}$ wave inversion returned to positivebiphasic $\mathrm{T}$ wave. It is believed that these conditions were induced by some phenomenon, such as myocardial stunning. MIBG scintigraphy obtained on the 48th postoperative day revealed that the reduction in uptake was much less than that obtained in the 5th postoperative day.

\section{Cardiographic Changes due to Sympathetic \\ Nervous Disorders}

Cardiographic giant negative $\mathrm{T}$ wave is known to appear not only with ischemic heart disease, but also with electrolyte abnormalities, autonervous disorders and various other causes. There have been various reports of QT-T alterations after injury of the sympathetic nervous system. Yanowitz et al showed that right stellate ganglion stimulation or left stellate ganglionectomy in dogs may produce such changes as Q-T interval prolongation and deeply inverted, prolonged T waves! Experimental evidence has indicated that stimulation of discrete hypothalamic areas will cause similar QT, ST, and T wave changes. Giant negative $\mathrm{T}$ wave associated with cerebrovascular accidents, especially subarachnoid hemorrhage, is also thought to be due to changes in sympathetic tone?

In this case, laminaplasty was performed at the C3-C5 spinal level and there was a possibility that sympathetic nervous damage occurred during the surgical procedure. The interference with the sympathetic nervous system was suspected as a possible cause for the drastic alteration of the $\mathrm{T}$ wave in the extensive leads without the presence of organic coronary stenosis.

One of the processes of sympathetic nervous injury that was suspected was that the tissue edema produced by the surgical procedure induced some damage in the sympathetic nervous system around the spine (eg, the postganglionic fibers of the ganglion cervicale superior, medium or inferior of the cardiac sympathetic nervous system).

The same electrocardiographic changes in the present case after cervical laminectomy have been reported in only one investigation as far as we know, and the authors concluded that the process of induction of the T- wave changes was that laminectomy may have irritated the grossopharyngeal and vagal nerve and initiated changes in the carotid sinus reflex, and that changed the balance of the autonomic nervous system activity?

\section{Uptake Reduction of MIBG due to Cardiac Sympathetic Nervous Disorders}

In patients with myocardial infarction, abnormal uptake reduction of both ${ }^{201} \mathrm{Tl}$ ) and ${ }^{123}$ I-MIBG has been noted in the areas perfused by occluded coronary vessels. Several studies have demonstrated that in patients with coronary vasospastic angina in whom coronary vasospasm was induced by intracoronary administration of acetylcholine, a regional uptake reduction of ${ }^{123}$ I-MIBG or a defect pattern was observed in the areas perfused by the vasospasminduced vessels, but neither reduced ${ }^{201} \mathrm{Tl}$ uptake nor defect pattern was detected 4

The regional reduction of uptake in MIBG is thought to reflect denervation of the myocardium resulting from severe ischemia5 MIBG uptake reduction has also been 
observed in direct denervation by stellate ganglionectomy in dogs. Dae et al reported that in dogs, MIBG was reduced relative to thallium in the posterior left ventricle in the left stellate ganglionectomized heart, whereas in the right stellate ganglionectomized heart, reduced MIBG occured in the anterior left ventricle, and a ${ }^{201} \mathrm{Tl}$ and ${ }^{123}$ I-MIBG uptake mismatch was detected in their study?

In our patient, the cardiac sympathetic denervation may have been a result of the cervical surgical procedure and, as a consequence of the denervation, reduction of MIBG uptake, which reflects cardiac sympathetic function, and ${ }^{201} \mathrm{Tl} /{ }^{123} \mathrm{I}-\mathrm{MIBG}$ uptake mismatch were provoked.

\section{Sympathetic Disorder and Myocardial Stunning}

Myocardial stunning, a prolonged but reversible myocardial contractile dysfunction, has been reported in patients with myocardial infarction, bypass surgery, hypertrophic cardiomyopathy, exercise-induced angina and vasospastic angina. Furthermore, some case reports have revealed that myocardial stunning is also observed in patients with cerebrovascular attack? and after electroconvulsive therapy8 The induction mechanism of myocardial stunning in these cases might be suspected to be through a sympathetic surge, but a definite conclusion has not been obtained yet. That invasion of the sympathetic nervous system could result in disordered cardiac function is itself a very interesting possibility. Further, some studies was demonstrated that electrical stimulation of the stellate ganglion in the dog causes not only marked electrocardiographic changes, but also subendocardial hemorrhage or focal myocardial necrosis?

In the present case, myocardial stunning occurred without evidence of myocardial ischemia or coronary vasospasm. We think this case suggests that sympathetic denervation itself induces myocardial stunning.

\section{References}

1. Yanowitz F, Preston JB, Abildscov JA: Functional distribution of right and left stellate innervation of the ventricles. Circ Res 1966; 28: $416-428$

2. Buruch GE, Meyers R, Abildskov JA: A new electrocardiographic pattern observed in cerebravascular accidents. Circulation 1954; 9: 719-723

3. Baur HR, Gobel FL, Picrach CA: Electrocardiographic changes after cervical laminectomy. Int J Cardiol 1981; 1: 37-40

4. Takano H, Nakamura T, Satou T, Umetani K, Watanabe A, Tamura $\mathrm{K}$, et al: Regional myocardial sympathetic dysinnervation in patient with coronary vasospasm. Am J Cardiol 1995; 75: 324-329

5. Inoue Y, Kugiyama K, Miyagi H, Ohgushi M, Tomiguchi S, Yasue $\mathrm{H}$, et al: Long-lasting abnormalities in cardiac sympathetic nervous system in patients with coronary spastic angina: Quantitative analysis with iodine 123 metaiodobenzylguanidine myocardial scintigraphy. Am Heart J 1997; 134: 112-118

6. Dae MW, O'Connell WJ, Botvinick EH, Ahearn T, Yee E, Munoz L, et al: Scintigraphic assessment of regional cardiac adrenergic innervation. Circulation 1989; 79: 634-644

7. Wang T-D, Wu C-C, Lee Y-T: Myocardial stunning after cerebral infarction. Int J Cardiol 1997; 58: 308-311

8. Zhu W-X, Olson DE, Karon BL, Tajik AJ: Myocardial stunning after electroconvulsive therapy. Ann Intern Med 1992; 117: 914-915

9. Klouda MA, Brynjolfsson G: Cardiotoxic effects of electrical stimulation of stimulation of stellate ganglia. Ann NY Acad Sci 1969; 156: $271-280$ 